\title{
Genetic Algorithm for Effective Optimization of Delay Performance in Wireless Sensor Networks
}

\author{
Saif Khalid Musluh ${ }^{\text {a }}$ \\ Alaa Abid Muslamb \\ Raid Abd Alreda Shekan \\ ${ }^{a}$ College of Biotechnology, University of Al-Qadisiyah,Iraq \\ ${ }^{b}$ College of computer science and IT, University of Al-Qadisiyah, Iraq \\ ${ }^{c}$ College of education for pure science, University of AL-Qadisiyah-Iraq \\ saif.khalid@qu.edu.iq_Alaa.abidmuslam@qu.edu.iq_pure.raed.abd@uobabylon.edu.iq
}

Submission date:- 11/11/2016 Acceptance date:- 12/12/2017 Publication date:- 16/12/2018

Keywords: Wireless Sensor Network, Genetic Algorithm, relay configuration, delay performance optimization.

\begin{abstract}
Wireless sensor networks (WSNs) play an important role in many real-world applications like surveillance. Wireless networks are also used to have data transfer. In such cases, there are problems with resourcececonstraintnednetworks. The problems include a delay in communication and reduction in Quality of Service (QoS). Topology control can solve this problem to some extent. However, the delay performance and QoS need to be improved further to support intended operations in wireless networks. When relay node concept is considered, it is possible to optimize performance in such networks. In this paper, we proposed a Genetic Algorithm (GA) based relay configuration for optimizing delay performance in WSN. Relay nodes compute optimal positions using the proposed algorithm so as to improve QoS and reduce delay as much as possible. We implemented the algorithm using NS2 simulations. The results revealed that the proposed approach is able to improve QoS, reduce delay besides improving network performance in terms of throughput, network capacity, and energy efficiency.
\end{abstract}

\section{1 - Introduction}

Wireless Sensor Network (WSN) is a network with a collection of sensor nodes that involve in data gathering and data transmission. The nodes in the network are resource constrained and they are vulnerable to various attacks. In this paper, we focus on the performance of the network in reducing delay and improving QoS in data transmission. The nodes in WSN can be configured in such a way that a node can act as a relay node. Thus the node can move to an optimal place which improves QoS and reduces delay in data transmission. This concept is known as relay configuration. Before looking at more details on this, a typical WSN is shown in Figure 1. 




Figure 1: A typical WSN [20]

As shown in Figure 1, it clear that WSN is deployed in target places and it can sense data and transfer it to sink node. The sink node can be accessed by intended users through the internet. In such scenarios, it is essential to ensure that the data transmission is not delayed and there is sufficient Quality of Service (QoS) in rendering required information. In the literature, many solutions came into existence [4]-[7]. However, it is relatively new to think about relay nodes and configuring them. The delay performance of wireless network one of the main factors that effected on network performance [9], [16], [17]. Therefore, it a hot area for researching. The researchers improve the delay performance based on many aspects. In [1], [2] improve the delay performance of the wireless network based on scheduling schemes of the network. [3] Combine both network protocols and network operations to optimize the delay. The control topology leads to control on the interference of network the result of that [5] use the control topology to enhance the delay. Meanwhile [8] depend on control topology and energy performance. [6] Optimize permeates of power, broadcast, interference, antenna, and reliability in order to enhance the delay. The mobility of network is a certain issue to enhance delay of the network [14],[15]. In this paper propose method based on Genetic Algorithm and relay configuration to enhance the delay performance of the wireless network.

In this paper we employed the idea of GA for best configuration of relay node. The relay node is the node which moves to an optimal position in order to transfer data faster and with efficiency. The node which relays data runs the proposed algorithm in order to have best possible decisions in terms of moving and rendering services. It finds the best place to move using GA and transfers data to reduce delay and improve QoS. The remainder of the paper is structured as follows. Section 2 provides a review of various methods available for dealing with delay optimization in wireless networks. Section 3 presents the proposed approach in delay optimization. Section 4 presents simulation results while section 5 provides conclusions and recommendations for future work.

\section{2- GA Based Relay Configuration for Performance Optimization}

This section provides the proposed relay configuration concept based on GA. GA is an evolutionary approach that has very important utility in real-world applications. In this paper, we used GA to optimize delay performance in wireless network and improve QoS. GA contains operators like crossover and mutation and work in evolutionary fashion until objective function is met. Thus it is found suitable for the delay optimization problem considered in this paper. When the nodes in the network in a typical WSN suffer from two problems such as increased delay and reduced QoS, it is essential to have a suitable solution. In this paper, we studied the relay configuration of nodes in the network in order to achieve delay performance optimization. One of the nodes acts as a relay node and moves to a different place in order to have efficient data transfer and reduce delay in communication. 


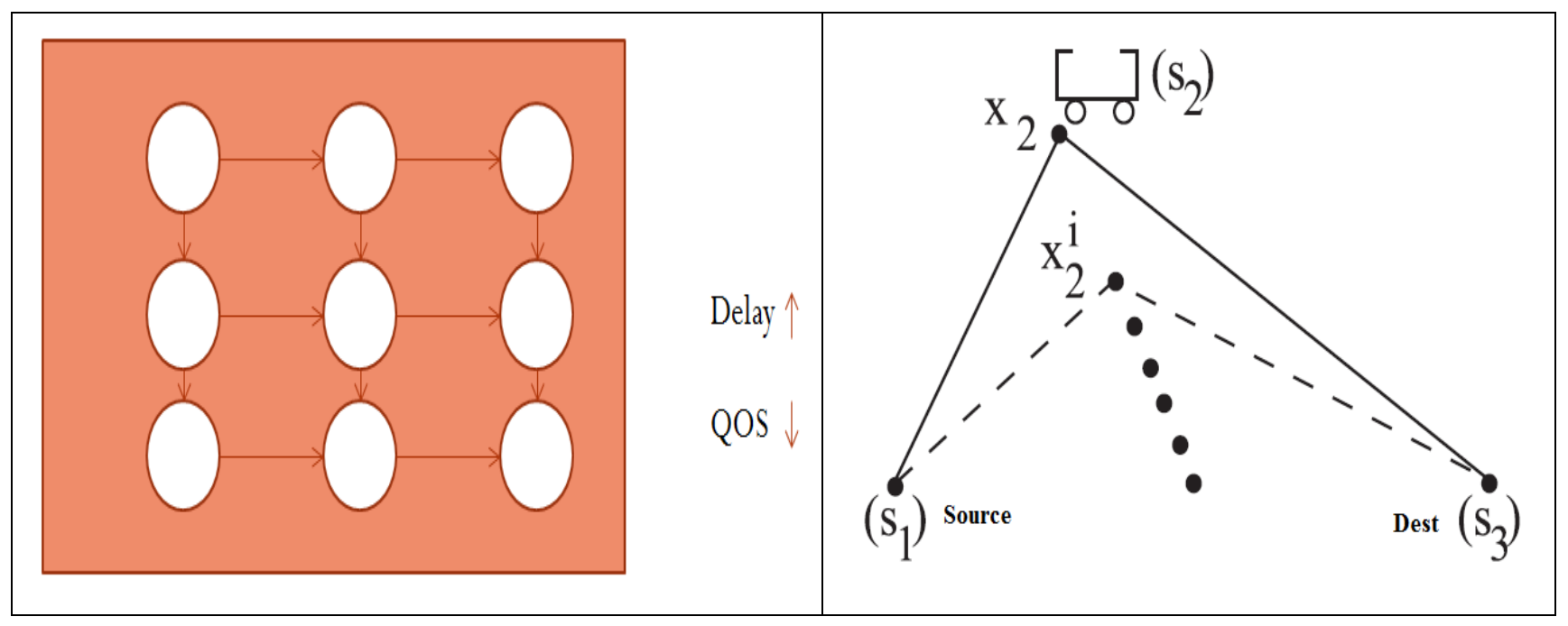

Figure 2: Shows problem (left) and solution in the form of GA based relay configuration

As shown in Figure 2, it is evident that there is delay problem with the existing network (left). Moreover, it is causing reduced QoS. In Figure 2 (right) relay configuration concept is presented. According to the proposed GA configuration of relay node, the node $\mathrm{x} 2$ moves to different location in order to transmit data. This is computed using an evolutionary approach (GA) based relay configuration. We proposed an algorithm to this effect and the algorithm takes care of optimization of delay performance and improves QoS in the network. The following Equations (1) and (2) are the underlying mechanisms in the relay configuration.

$c(\langle E, U\rangle)=\sum_{\left(s_{i}, s_{j}\right)} a m_{i}+b\left\|\mu_{i}-\mu_{j}\right\| 2 m_{i}+k\left\|o_{i}-\mu_{i}\right\|$

$c_{i}(U)=k\left\|\mu_{i}-o_{i}\right\|+a m+b\left\|\mu_{i+1}-\mu_{i}\right\| 2 m$

These equations provide cost-related configurations that are used to make well-informed decisions. The proposed algorithm makes use of them effectively. More details on these concepts are available in [18].



Algorithm 1: Genetic Algorithm Based Relay configuration 
The algorithm considers both delay and interference with the network and performs optimization. The algorithm is executed by the relay node which uses GA based approach in order to optimize relay configuration thus reduce delay and improve QoS. The algorithm is implemented using NS2 simulations. The work of the paper is divided into WSN construction, controlling topology, GA based relay configuration and achieving delay performance. WSN is created using NS2 simulation. The network is a collection of nodes that are configured to gather data and transfer data. The basic WSN network is built in order to demonstrate proof of the concept in this paper. The network is used to show delay optimization, QoS enhancement and other performance metrics like throughput.

Topology control is involved in the form of adjusting nodes in order to reduce interference and improve delay performance. This can lead to QoS in wireless network. The proposed algorithm analyzes delay and takes necessary steps to reduce delay. Especially it considers relay nodes moving places for better data transfer. The evolutionary GA based approach for relay configuration is employed as it can make use of operators of GA for best possible solution. Delay and interference thresholds are used to make good decisions while computing benefits of moving places with respect to delay node. The relay configuration is computed effectively by considering position of the node, source, and destination based on the data to be transferred. Node movement is determined using the proposed algorithm for gaining maximum benefits. The mobility cost and data transfer cost are considered while making decisions.

\section{3 - Simulation Results}

A simulation study with NS2 is made to demonstrate the concept of relay configuration using GA with underlying operators. The proposed algorithm is implanted using simulations. The observations are made in terms of delay, throughput, and network capacity and so on. The simulation environment used in this paper is shown in Table 1 .

Table 1: Simulation environment

\begin{tabular}{|l|l|l|}
\hline S.No & Parameter Type & Parameter Value \\
\hline 1 & Channel Type & Wireless Channel \\
\hline 2 & Radio-Propagation & Propagation/TwoRayGround \\
\hline 3 & Network Interface & WirelessPhy \\
\hline 4 & Interface Queue Type & DropTail \\
\hline 5 & Antenna Model & OmniAntenna \\
\hline 6 & Interface Queue Length & 50 \\
\hline 7 & Routing Protocol & AODV \\
\hline 8 & CTSThreshold & 2000 \\
\hline 9 & RTSThreshold & 5000 \\
\hline 10 & basicRate & $1 \mathrm{MB}$ \\
\hline 11 & dataRate & $5 \mathrm{MB}$ \\
\hline
\end{tabular}

The simulation environment considered many parameters. They include channel type, radio-propagation, network interface, interface queue type, antenna model, routing protocol and so on. The routing protocol used for simulations is AODV. GA based relay configuration algorithm is employed to improve delay performance while transferring data. 


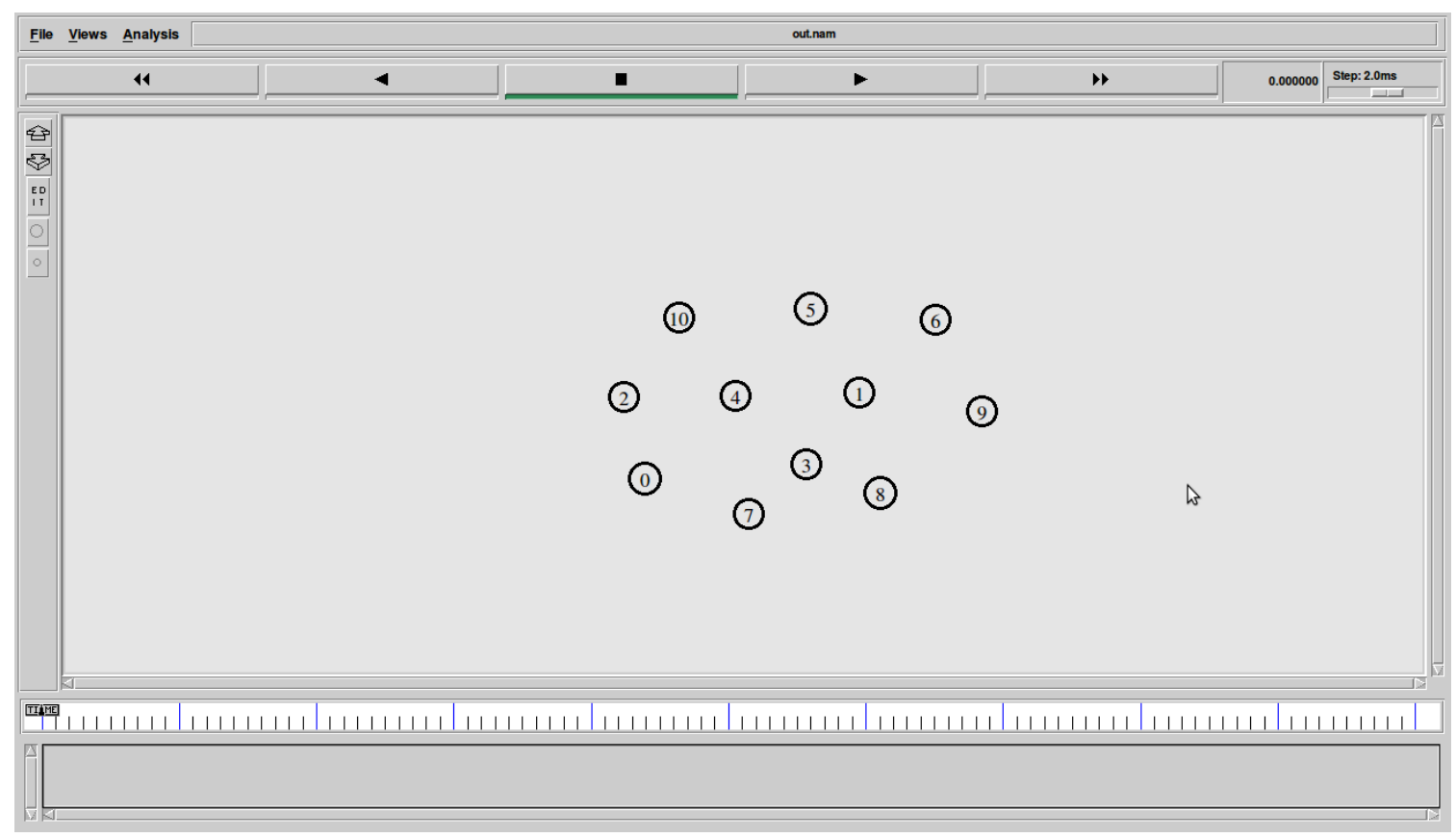

Figure 3: A typical WSN

As presented in Figure 3, it is evident that the wireless network is constructed with many nodes. The nodes are placed in the network with standard settings as presented in Table 1. The simulation is aimed at showing relay node positioning and its configuration using GA based algorithm.

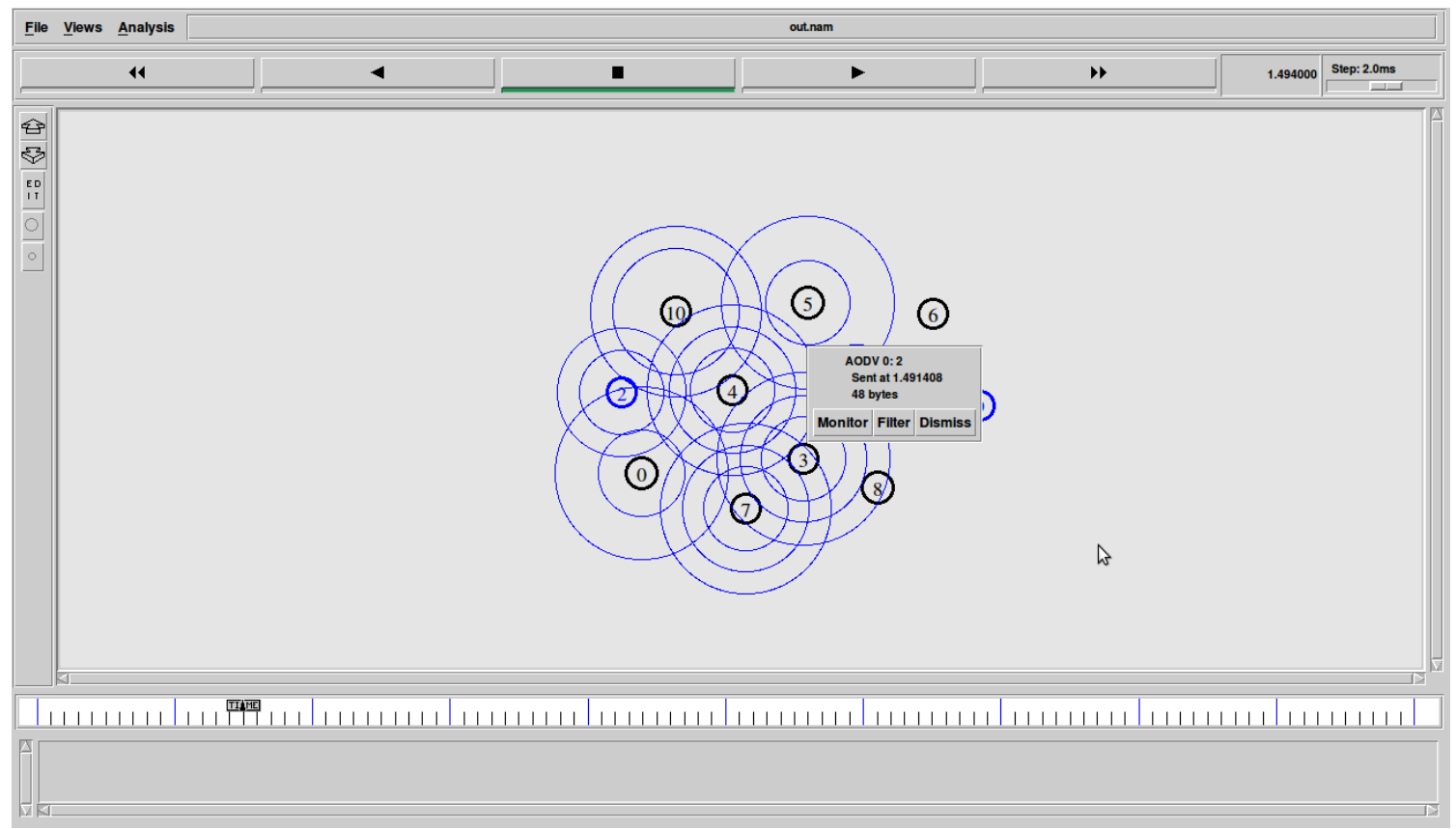

Figure 3: Wireless network with AODV protocol propagation

As presented in Figure 4, it is evident that the proposed in wireless network that is AODV started with intended functionality. The protocol propagation is shown that reflects initial operations such as protocol handshaking. 


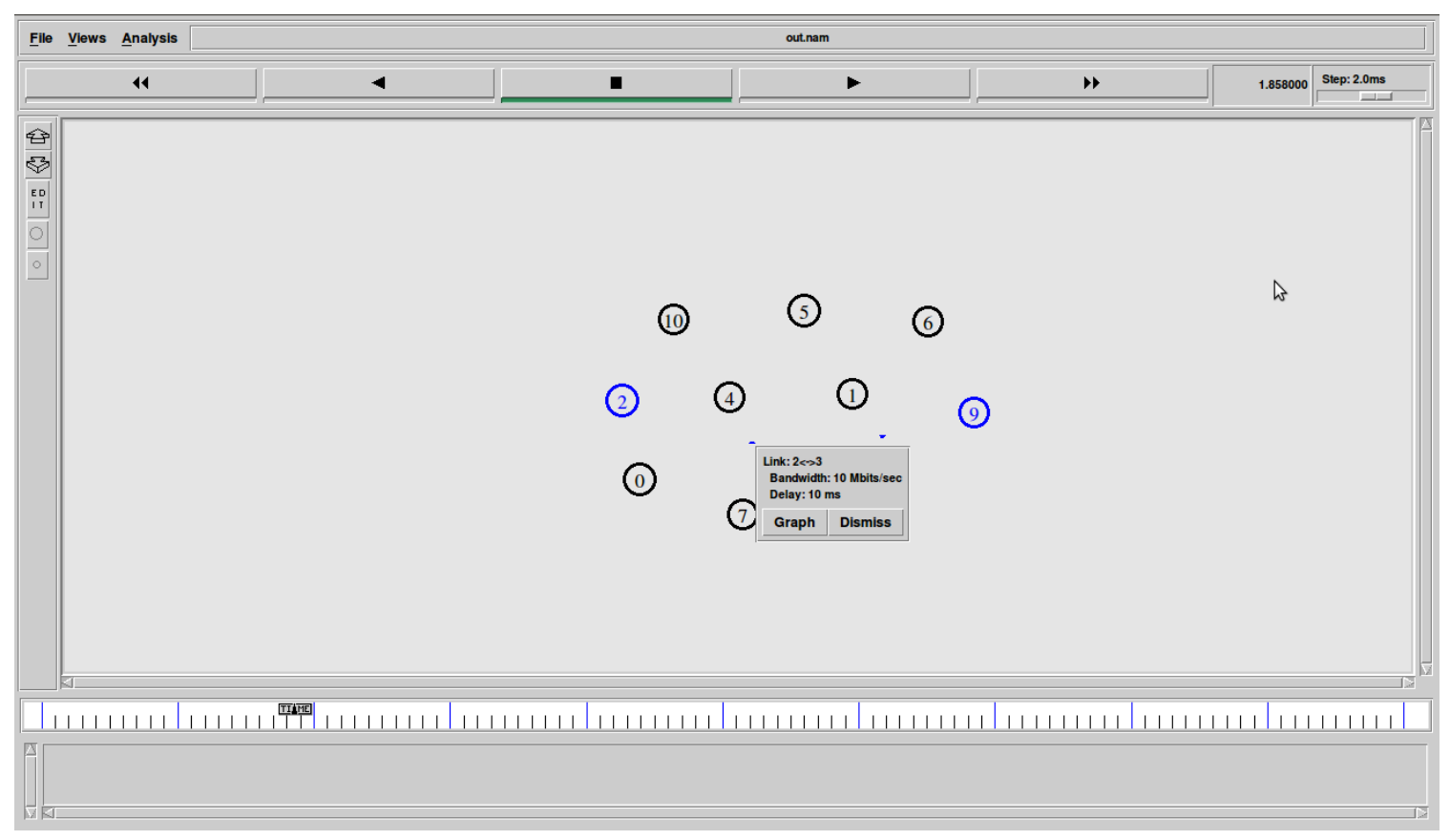

Figure 5: Shows packet interval, bandwidth, and link between nodes

As presented in Figure 5, it is evident that the proposed protocol shown the communication scenario between nodes. The details shown in the simulation include bandwidth, packet interval, and delay performance.

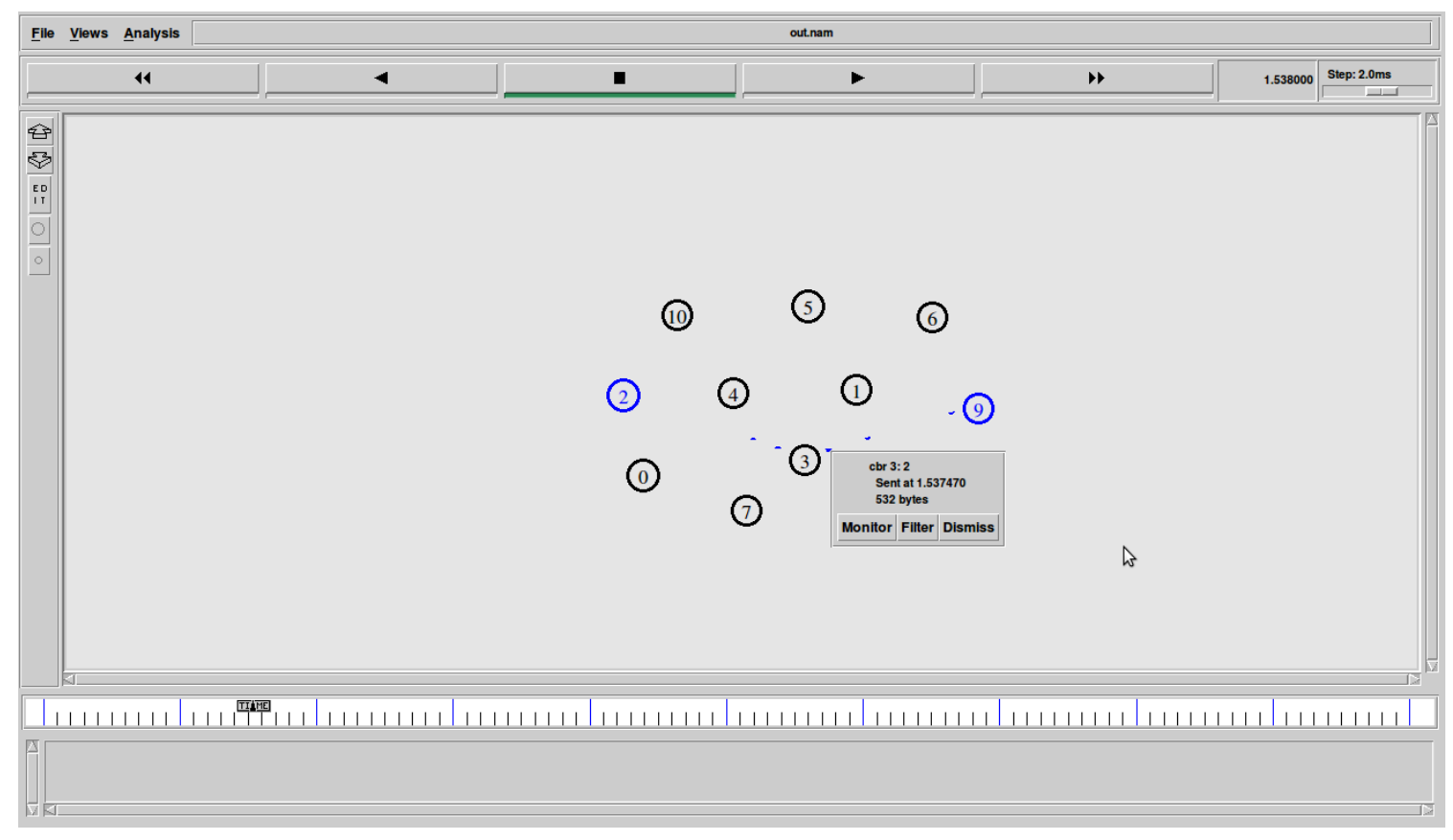

Figure 6: Shows packet transmission between source and destination nodes

As presented in Figure 6, it is evident that the simulation shows flow of packets between pre-defined source and destination. The CBR model of packets is flown between source and destination. 


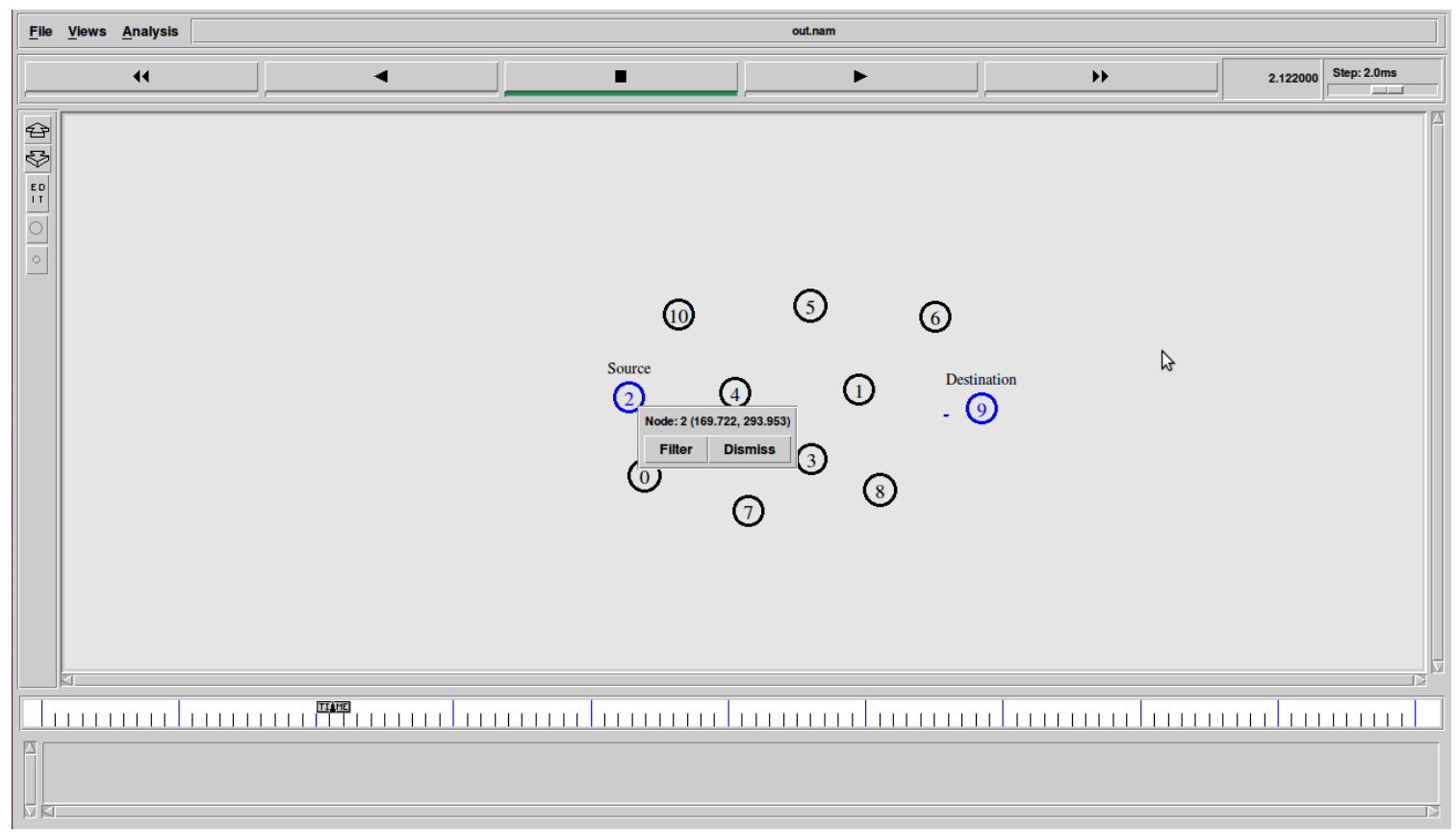

Figure 7: Demonstrates node movement and relay

As presented in Figure 7, it is evident that the relay node is moving to different location to have an optimized data transfer. The efficiency of GA based evolutionary algorithm is evident in the simulation as the relay node is able to compute optimal movement for better data transfer performance.

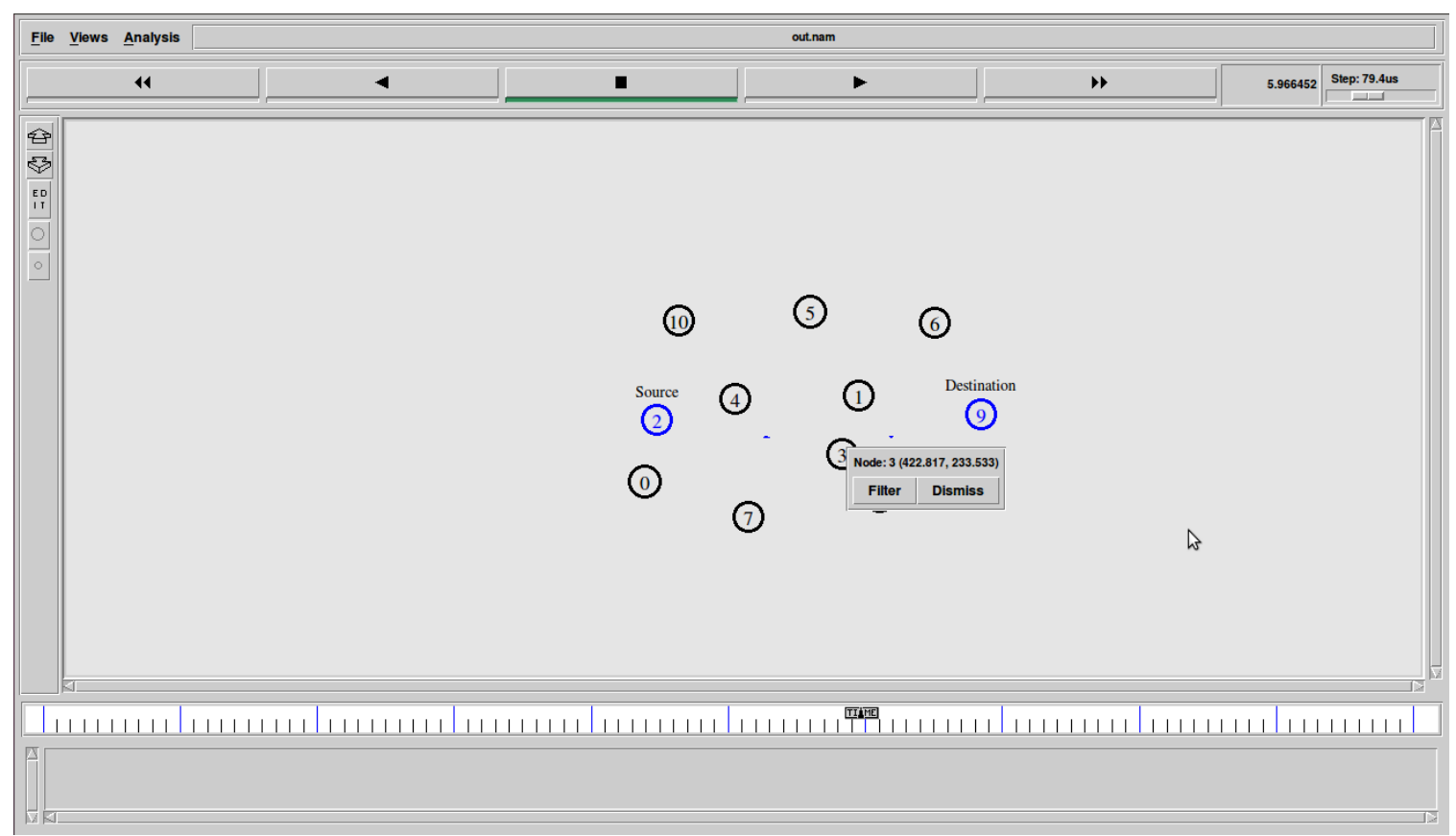

Figure 8: Shown Node 3 moving with relay configuration in place

As presented in Figure 8, the simulation shows the movement of an intermediate node according to the GA based relay configuration algorithm before sending data to destination. 


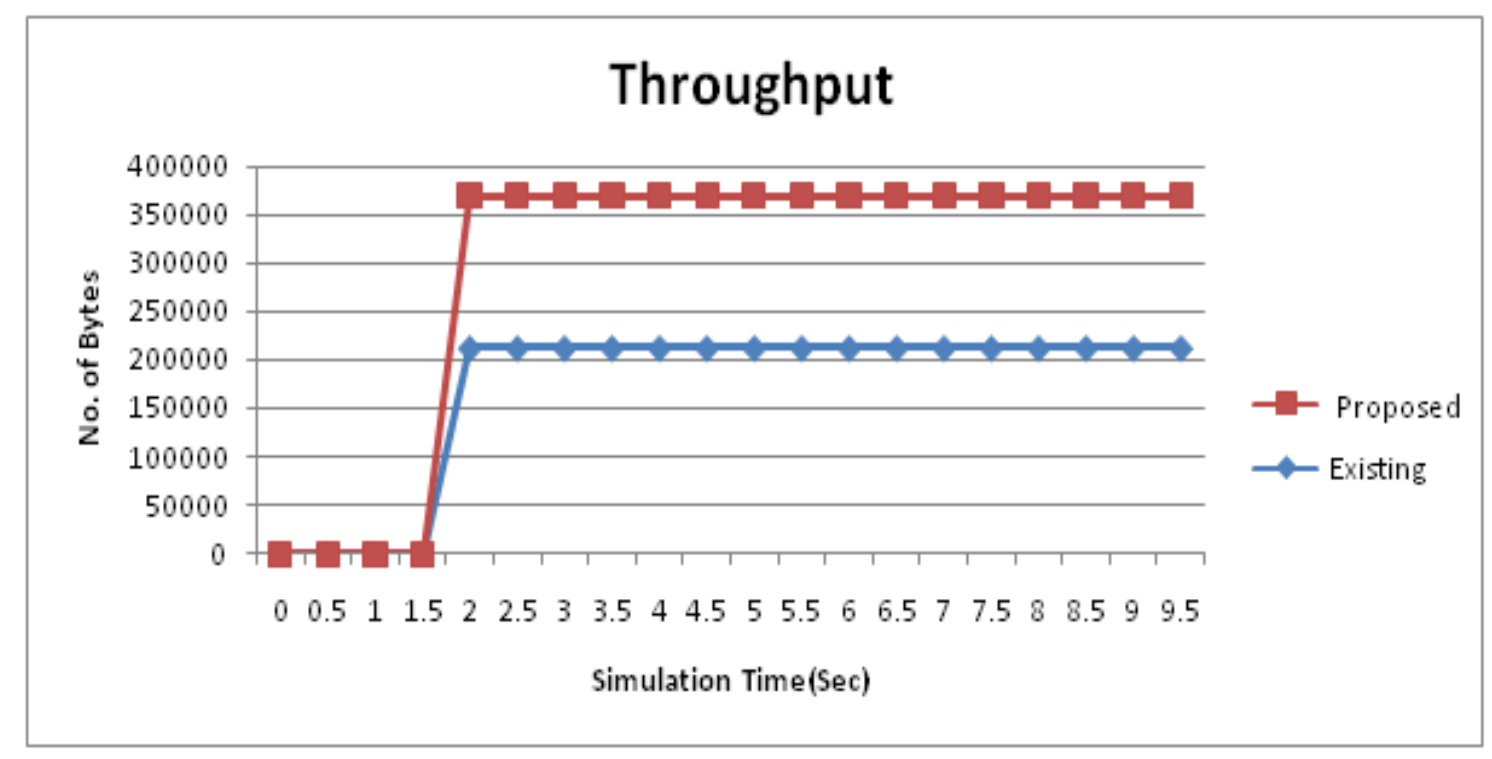

Figure 9: Throughput performance

As presented in Figure 9, it is clear that simulation time and throughput are plotted in horizontal and vertical axes respectively. The results show that the trouped performance of the proposed system is better than that of existing. The rationale behind this is the utility of proposed GA based relay algorithm for reducing delay.

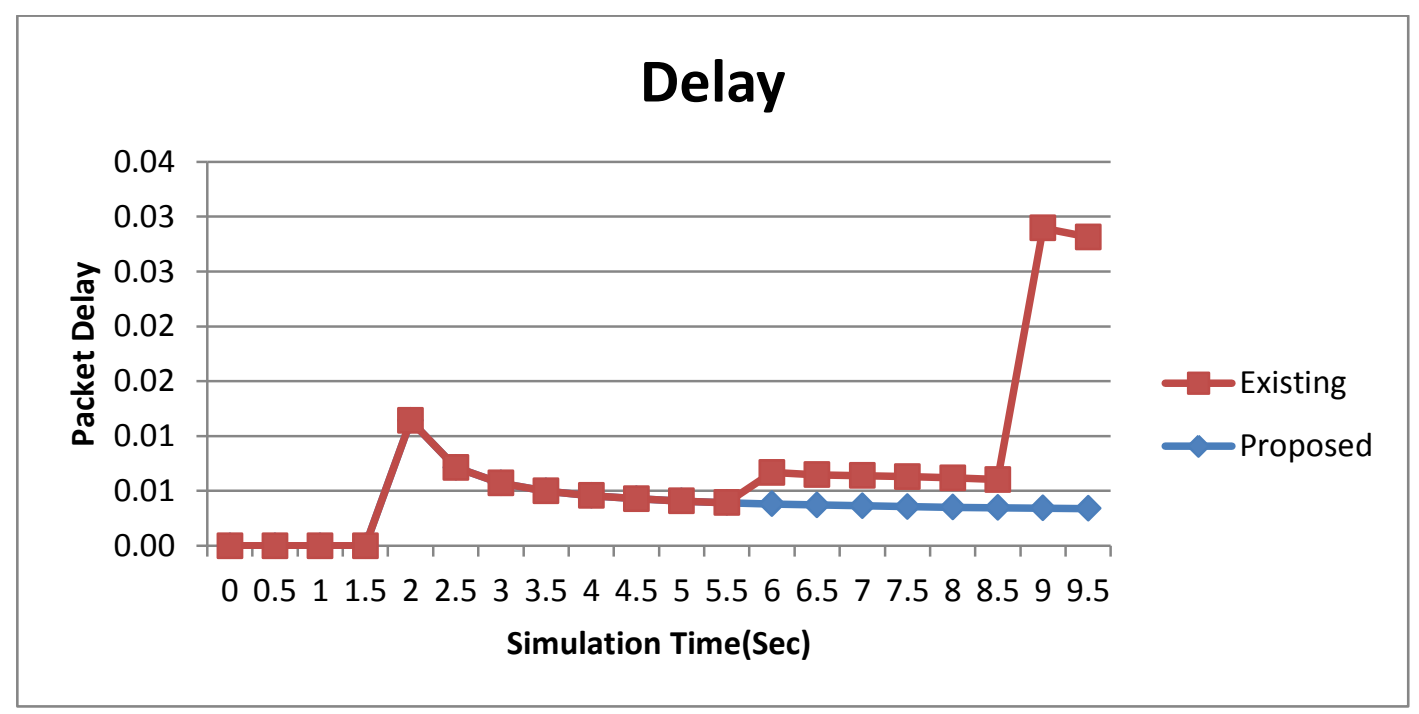

Figure 10: Delay performance comparison

As presented in Figure 10, it is clear that the performance of the proposed approach is better as it shows less delay. As simulation time goes on the delay is decreased in the proposed system. The existing system shows the same trend but its performance is less than that of the proposed system. 


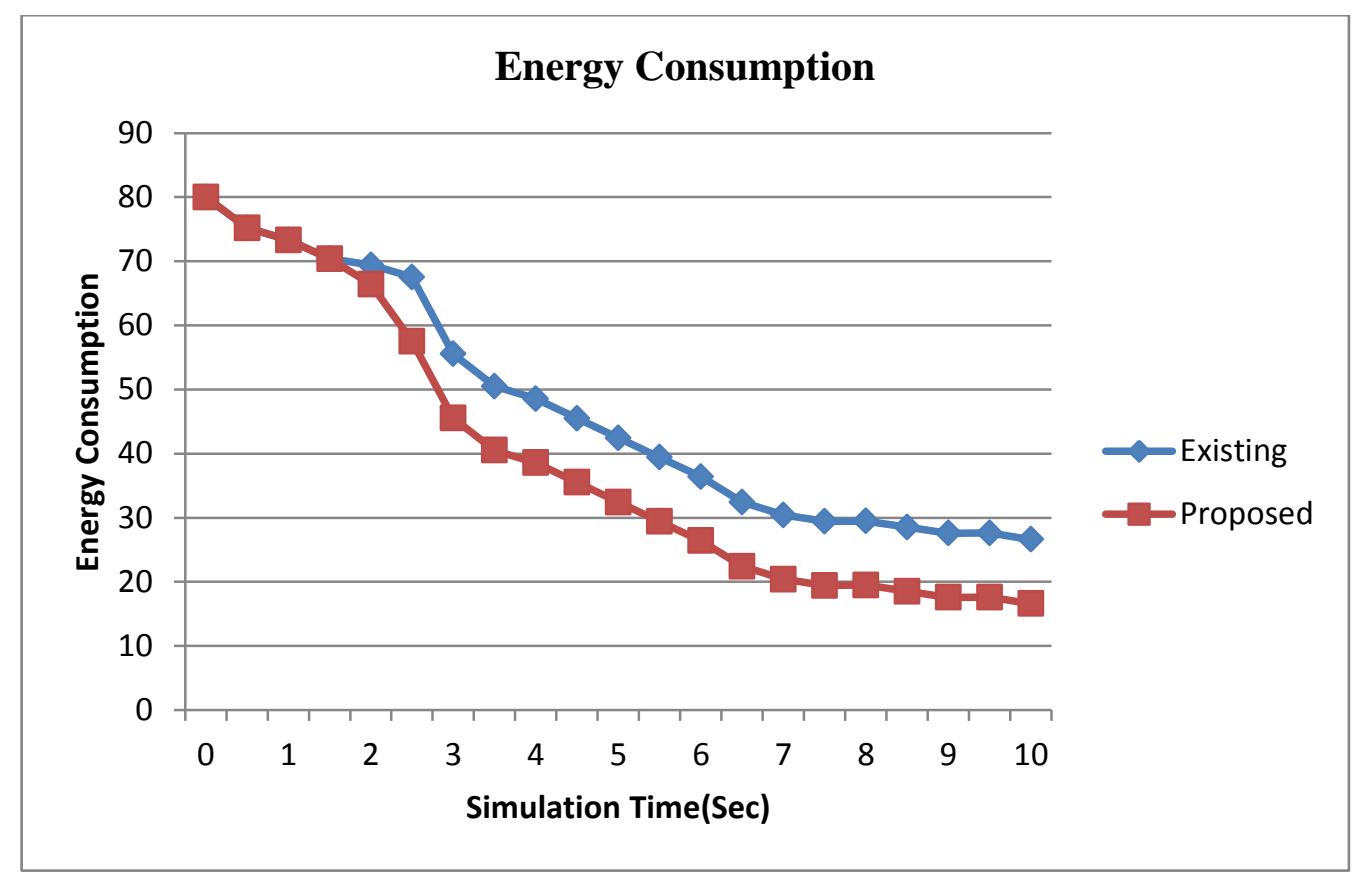

Figure 11: Energy consumption

As presented in Figure 11, it is clear that the energy consumption of the proposed system is reduced. As simulation time is increased, the energy consumption showed improved performance. In other words, it can be said that the residual energy is more in the proposed system.

\section{4 - Conclusions and FUTURE WORK}

Wireless sensor networks provide means of collecting data from remote places. Even in hostile environments, it is possible to obtain surveillance information. Interference and delay are the two problems of WSN focused in this paper. When these two parameters are considered, it is essential to have an approach that can handle topology and node mobility. To ensure delay performance in such networks, it is required to have an efficient approach. In this paper, we employed GA based mobile relay configuration algorithm to optimize delay performance. The approach considers one of the noses as relay node which is responsible to move to a different place if needed in order to relay data. Thus it can improve performance in data transmission. The proposed algorithm in this paper makes use of GA operators and objective function in order to have an evolutionary mechanism to determine the movement of relay node for optimized performance. We implemented the algorithm using N2 simulations. The results showed that the proposed approach is able to provide better performance in terms of network capacity, delay performance, energy efficiency and throughput.

\section{References}

[1] A. Muqattach and M. M. Krunz, "A distributed transmission power control protocol for mobile ad hoc networks," IEEE Trans. Mobile Comput., vol. 3, no 2, pp. 113-128, Apr.-Jun. 2004.

[2]C. F. Chou and H. P. Suen, "Topology-control-based QoS routing (TLQR) in wireless ad hoc networks," in Proc. IEEE 17th Int. Symp. Pers., Indoor Mobile Radio Commun., 2006, pp. 1-5.

[3]D. M. Blough, M. Leoncini, G. Resta, and P. Santi, "The k-neighbors approach to interference bounded and symmetric topology control in ad hoc networks," IEEE Trans. Mobile Comput., vol. 5, no. 9, pp. 1267-1282, Sep. 2006.

[4]D. Y. Xue and E. Ekici, "Delay-guaranteed cross-layer scheduling in multihop wireless networks,"IEEE/ACM Trans. Netw., vol. 21, no. 6, pp. 1696-1707, Dec. 2013.

[5]El-Moukaddem, F, Torng, E., Xing, G. (2013). Mobile Relay Configuraion in Data-Intensive Wireless Sensor Networks. IEEE, p261-273.

[6]F. Xie, X. M. Zhang, J. F. Guo, and G. L. Chen, "A delay oriented adaptive routing protocol for mobile ad hoc networks, (in Chinese with English abstract,” J. Softw., vol. 16, no. 9, pp. 1661-1667, 2005.

[7]J. Kim and Y. Kwon, “Interference-aware topology control for low rate wireless personal area networks,"IEEE Trans. Consume. Electron. vol. 55, no. 1, pp. 97-104, Feb. 2009. 
[8]J. Mammen and D. Shah, "Throughput and delay in random wireless networks with restricted mobility,” IEEE Trans. Inf. Theory, vol. 53, no. 3, pp. 1108-1116, Mar 2007.

[9]J. Tang, G. L. Xue, and W. Y. Zhang, "Interference-aware topology control and QoS routing in multi-channel wireless mesh networks," in Proc. ACM 6th Int. Symp. Mobile Ad Hoc Netw. Comput., 2005, pp. 68-77.

[10]M. Burkhart, P. von Rickenbach, R. Wattenhofer, and A. Zollinger, "Does topology control reduce interference," in Proc. ACM 5th Int. Symp. Mobile Ad Hoc Netw.Comput., 2004, pp. 9-19.

[11]M. Kadivar, M. E. Shiri, and M. Dahghan, "Distributed topology control algorithm based on one- and two-hop neighbors' information for ad hoc networks," Comput. Commun., vol. 32, no. 2, pp. 368-375, 2009.

[12]P. Santi, "Topology control in wireless ad hoc and sensor networks," ACM Compute. Surv., vol. 37, no. 2, pp. 164194, 2005.

[13]Q. Zhang and Y. Q. Zhang, “Cross-layer design for QoS support in multichip wireless networks,” Proc. IEEE, vol. 96, no. 1, pp. 64-76, Jan. 2008.

[14]R. G. Li and A. Eryilmaz, "Scheduling for end-to-end deadline constrained traffic with reliability requirements in multihop networks," IEEE/ACM Trans. Netw., vol. 20, no. 5, pp. 1649-1663, Oct. 2012.

[15]S. C. Wang, D. S. Wei, and S.-Y. Kuo, "An SPT-based topology control algorithm for wireless ad hoc networks," Comput. Commun., vol. 29, no. 16, pp. 3092-3103, 2007.

[16]X. H. Jia, D. Y. Li, and D. Z. Du, "QoS topology control in ad hoc wireless networks," in Proc. IEEE Conf. Comput. Commun., 2004, pp. 1264-1272.

[17]X. M. Zhang, F. F. Zou, E. B. Wang, and D. K. Sung, "Exploring the dynamic nature of mobile nodes for predicting route lifetime in mobile ad hoc networks," IEEE Trans. Veh. Technol., vol. 59, no. 3, pp. 1567-1572, Mar. 2010.

[18]X. Zhu, P. Li, Y. Fang, and Y. Wang, "Throughput, delay, and mobility in wireless ad hoc networks," in Proc. IEEE Conf. Comput. Commun., 2010, pp. 1-9.

[19]Y .Perkins, Charles E., et al. "Performance comparison of two on-demand routing protocols for ad hoc networks." IEEE Personal communications 8.1 (2001): 16-28.

[20]Z. Karl, Holger, and Andreas Willig. Protocols and architectures for wireless sensor networks. John Wiley \& Sons, 2007.

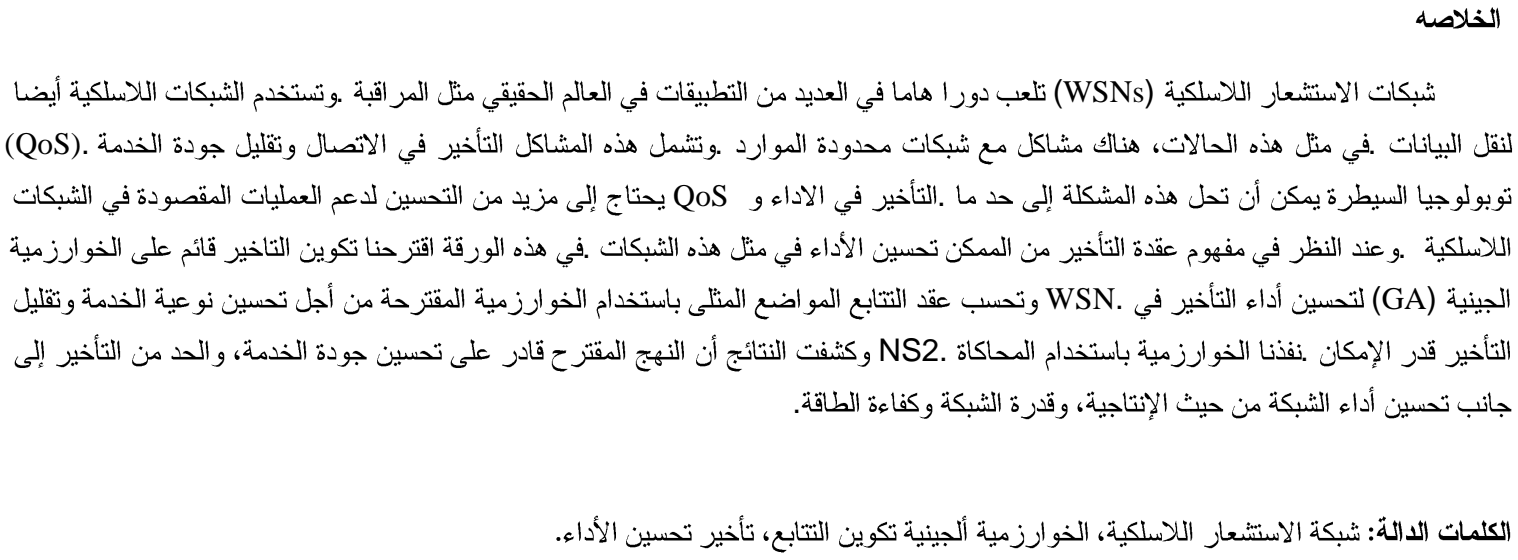

